VIII ${ }^{\text {èmes }}$ Journées Nationales Génie Civil - Génie Côtier, Compiègne, 7-9 septembre 2004

\title{
Démarche de caractérisation de la zone non saturée d'une friche industrielle en vue de modéliser le flux de polluant transitant vers un système fluvial.
}

\author{
Picot Géraldine ${ }^{(\mathrm{a}, \mathrm{b})}$, Bernard Fabrice ${ }^{(\mathrm{a})}$, Abriak Nor-Edine ${ }^{(\mathrm{a})}$. \\ ${ }^{(a)}$ Ecole des Mines de Douai, Département Génie Civil, \\ 941, rue Charles Bourseul, BP 838, 59508 DOUAI cedex, France, \\ ${ }^{(b)}$ Laboratoire Artois de Mécanique, Thermique et Instrumentation, \\ Faculté des Sciences Appliquées, Technoparc Futura, 62400 Béthune, France.
}

\section{Résumé}

Le travail abordé ici s’inscrit dans une étude globale de mise au point d'outils de caractérisation et de modélisation de flux de polluant transporté par l'eau (approche quantitative) transitant dans la zone non saturée d'un sol. Le travail présente, dans un premier temps, la démarche d'étude suivie pour caractériser et modéliser les écoulements pollués dans un sol non saturé provenant de sites pollués. Puis dans un deuxième temps, la réalisation d'une des étapes de la démarche : la validation de l'outil de modélisation choisi, travaillant par éléments finis, sur une expérience d’infiltration de référence.

\begin{abstract}
$\underline{\text { Abstract }}$
The general context of this study is the development of tools for characterisation and modelling of contaminated flow (quantitative approach) in unsaturated porous soil. First we present the working method used for such characterisation and modelling. Then we focus on a specific step of this methodology: the validation of the chosen finite element method software (Feflow) on an infiltration experiment.
\end{abstract}

Mots-clés: écoulements, zone non saturée, site pollué, modélisation.

Keywords: flows, unsaturated zone, coking plant, modelling.

\section{Introduction}

Au cours du temps, l'accumulation de déchets et de substances déposés ou déversés s'est avérée néfaste pour le milieu naturel environnant. En effet, les précipitations arrivant au sol se décomposent en (Figure 1$)^{1}$ : une partie qui ruisselle en emportant une part des contaminants présents à la surface du sol (R), et une partie qui s'infiltre (I) dans la zone non saturée du sol (si les conditions du sol le permettent). Les eaux qui s’infiltrent, peuvent soit se déplacer latéralement dans la zone non saturée (Qs) jusqu'à atteindre le système fluvial à proximité, où peut s'observer une émergence de flux pollués $(\mathrm{Em})$; soit s'infiltrer jusqu'à la nappe superficielle, par déplacement vertical (Rg) et ainsi la contaminer. Si la configuration hydrogéologique de la nappe permet la ré-alimentation du système fluvial (Qg), ce dernier peut également subir une contamination.

Ces différents cas montrent l'importance d'étudier la zone non saturée du sol et ses écoulements afin d'estimer le flux de polluant transporté par l'eau y transitant vers un système fluvial. 

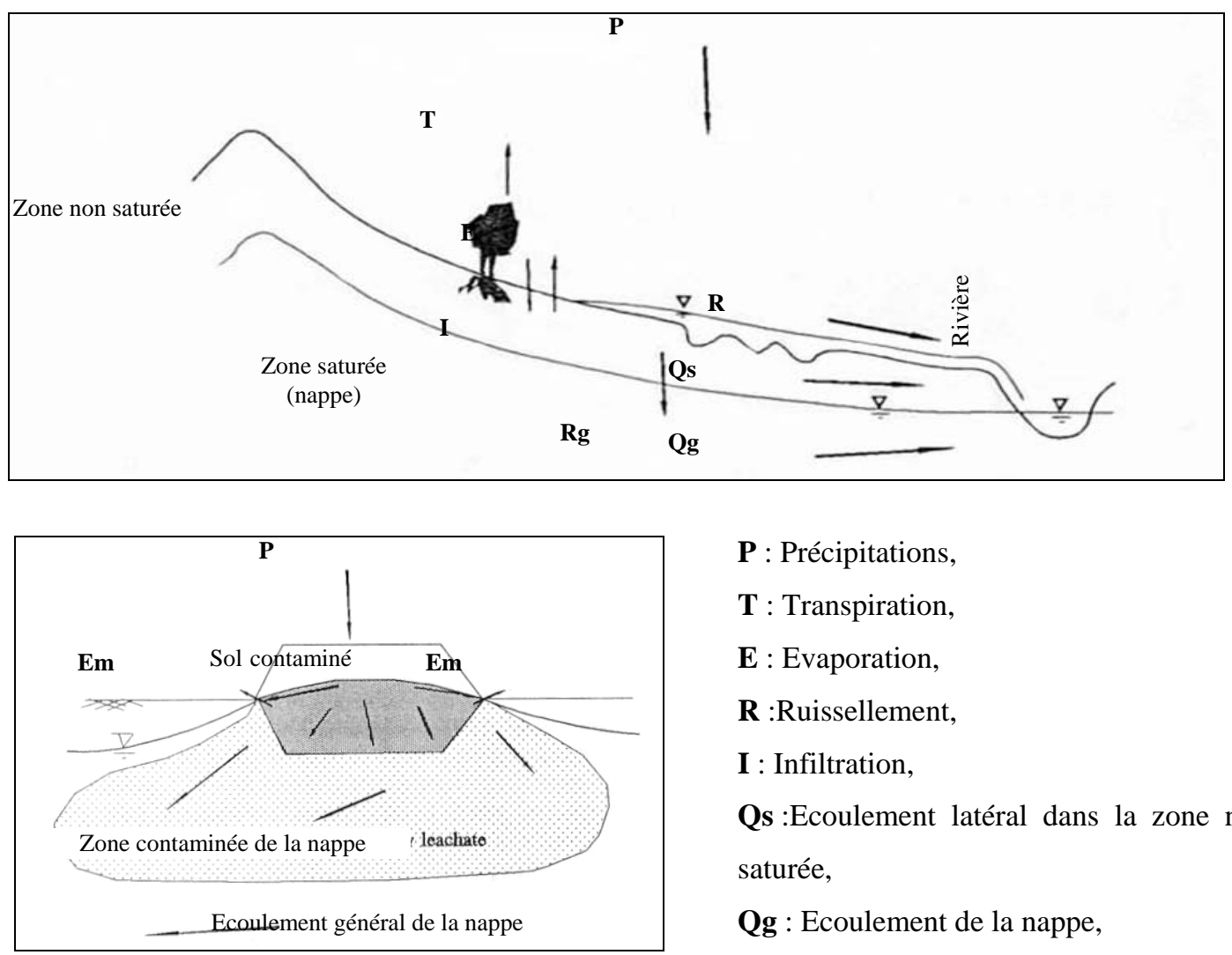

P : Précipitations,

$\mathbf{T}$ : Transpiration,

E : Evaporation,

$\mathbf{R}$ :Ruissellement,

I : Infiltration,

Qs :Ecoulement latéral dans la zone non saturée,

Qg : Ecoulement de la nappe,

Rg : Recharge de la nappe,

Em : Emergence de flux contaminé,

Figure 1. Principaux écoulements arrivant dans un système fluvial.

Cependant des incertitudes mettent en balance la caractérisation de l'impact réel d'une pollution sur son environnement. Il existe, en effet, encore des lacunes au niveau des connaissances actuelles sur les polluants eux-mêmes et sur les écoulements dans le sol. La modélisation peut apparaître comme un outil intéressant dans la caractérisation de ces écoulements dans un sol ou dans la prise de décision après simulation. Il est alors nécessaire de connaître les différentes méthodes et champs d'application de ces outils pour ne pas détourner l'information des données réelles initiales. Nous abordons dans un premier temps la démarche de travail suivie pour caractériser et modéliser les écoulements pollués dans un sol non saturé. Puis dans un deuxième temps, est présentée une des étapes de la démarche réalisée: la validation de l'outil de modélisation choisi sur une expérience d'infiltration de référence.

\section{Démarche de travail}

La caractérisation et la modélisation des écoulements dans un sol poreux non saturé au droit d'un site pollué quel qu'il soit, se réalisent en suivant plusieurs étapes de travail chronologiques représentées sur la figure 2. Cette démarche regroupe quatre grands points : le recueil des informations disponibles sur le site étudié, l'identification du(des) polluant(s) à suivre, un complément de données et enfin la modélisation-simulation du transport du(des) polluant(s).

La première étape de cette démarche, "Analyse de l'existant du site», reprend toutes les informations disponibles sur le site étudié tant au niveau des activités exercées sur le site 
qu'au niveau de son environnement en surface et en profondeur. L'étude de l' «exploitation» permet de connaître les différentes activités qui se sont succédées sur le site et de ce fait, d'en déduire les substances néfastes pour l'environnement à étudier. Le recueil des «données de surface» rassemble les informations de localisation géographique et de géométrie du site étudié, le contexte topographique et hydrologique de surface, ceci permettant, lors des étapes de modélisation, de délimiter le domaine et de positionner certaines conditions aux limites (cours d'eau, ...).

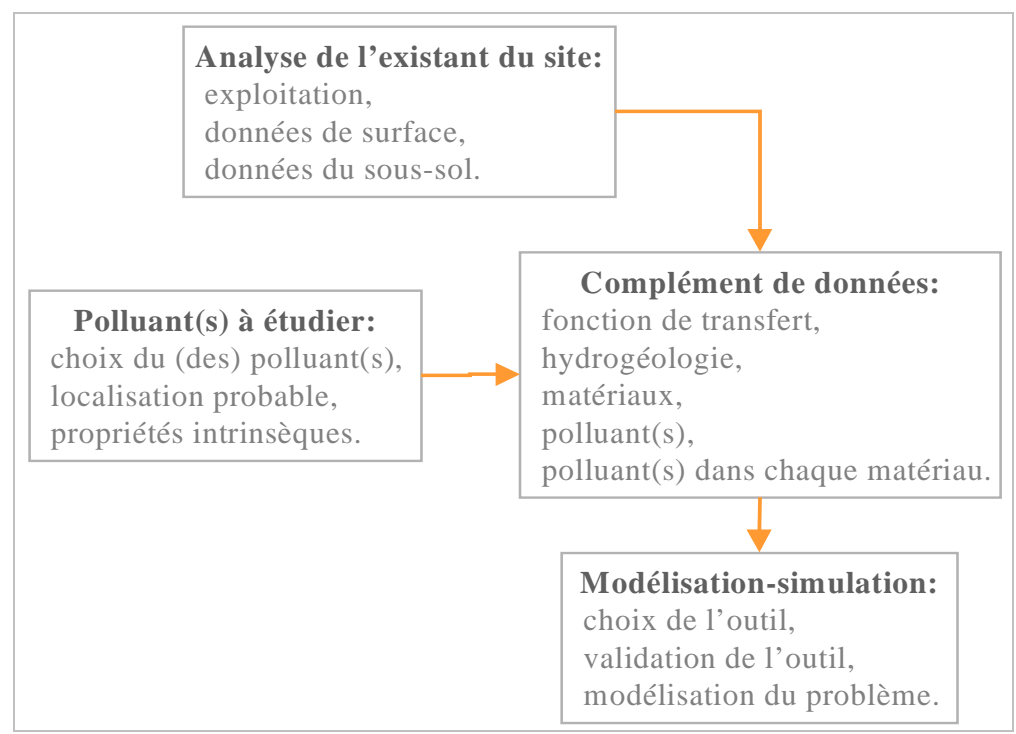

Figure 2. Démarche globale de travail.

Enfin le recueil des «données du sous-sol» (pédologiques, géologiques, hydrogéologiques, géotechniques et réseaux enterrés) donne un aperçu des terrains constituant le sous-sol et leur stratification, de l'épaisseur de la zone non saturée du sol à identifier ainsi que la localisation des zones dans lesquelles les prélèvements de sols et d'eau de nappe seront réalisables. Cette première étape révèle alors le contexte général dans lequel évolue le sous-sol du site étudié qui doit être confirmé par la troisième étape : «complément de données». Avant cela, il est d'abord nécessaire d'identifier le ou les polluant(s) à suivre dans cette étude.

Ce choix est tout d'abord lié aux différentes activités et productions exercées sur le site étudié (cf. Analyse de l'existant), puis guidé par l'impact sur l'environnement que peut avoir le(les) polluant(s). La localisation de la source polluante peut être déduite de la localisation de certaines infrastructures ou dépôts. Une fois ce choix effectué, il est nécessaire de s'informer sur les propriétés intrinsèques de chaque polluant pris en compte dans l'étude pour connaître l'état sous lequel il se trouve dans le sol et les eaux (gaz, liquide, solide) et dans quelles conditions il peut changer d'état. Les propriétés intrinsèques rassemblent donc les paramètres tels que : densité, pression vapeur, solubilité, constante d'Henry, température de fusion et d'ébullition, valeurs guides de l'organisation mondiale pour la santé et néerlandaises (niveau d'intervention pour la protection des eaux souterraines ou des sols), etc. Le choix du(des) polluant(s) à étudier est donc analysé dans cette deuxième étape de la démarche, mais il doit être confirmé sur le site étudié par analyses des eaux des nappes souterraines et des sols prélevés.

La troisième étape consiste à définir les modèles mathématiques (choix et paramétrages) à utiliser pour modéliser le transport des polluants dans la zone non saturée du sol, puis à réaliser des investigations de terrains pour compléter et confirmer les informations recueillies lors des étapes précédentes. Tout d'abord, les modèles mathématiques doivent refléter les 
conditions de travail fixées, c'est à dire qu'ils doivent prendre en compte les écoulements en milieu poreux non saturé en régime transitoire (équation de Richards ${ }^{2}$, éq.(1)), puis le transfert de polluant (éq. (2)), phénomènes décrits par les équations suivantes :

$$
\begin{aligned}
& \operatorname{div}(K(h) \operatorname{grad}(H))=C(h) \frac{\partial h}{\partial t} \\
& \frac{\partial(C)}{\partial t}=\operatorname{div}(\operatorname{Dgrad} C-\vec{q} C)+Q
\end{aligned}
$$

avec t le temps, $\mathrm{H}$ la charge hydraulique, h le potentiel matriciel, $\mathrm{C}$ la concentration du polluant, D la dispersion hydrodynamique, $\mathrm{C}(\mathrm{h})=\mathrm{d} \theta / \mathrm{dh}$ la capacité d'humectation, $\mathrm{K}(\mathrm{h})$ la relation conductivité hydraulique-charge qui est exprimée par des modèles empiriques tels que Van Genutchen, Haverkamp, etc, et enfin Q qui exprime les phénomènes de sorption existant entre le polluant et son environnement.

Pour appliquer les formulations choisies, il est nécessaire de lister l'ensemble des paramètres nécessaires afin de réaliser les essais de caractérisation adéquats sur le matériau à travers lequel transitent les écoulements ainsi que sur les polluants considérés. C’est pourquoi des investigations sont à réaliser sur le site étudié selon quatre axes :

- caractérisation hydrogéologique : à l'aide de piézomètres implantés jusqu’à la nappe phréatique et de mesures de niveau d'eau, l'épaisseur de la zone non saturée du sol à étudier peut être définie,

- caractérisation des matériaux: à l'aide de carottages et de sondages, la configuration 3D des terrains composant la zone non saturée du sol est améliorée puis des prélèvements et analyses en laboratoire ou in situ permettent une caractérisation des paramètres nécessaires (état de saturation, porosité, conductivité hydraulique, ...),

- caractérisation du(des) polluant(s) : les prélèvements et analyses de sols et d'eaux de nappe confirment le choix du polluant à étudier sur le site et préciseront sa localisation sur le terrain,

- caractérisation polluant-matériau: à l'aide d'essais en laboratoire, le comportement du polluant vis à vis de son environnement est quantifié et les phénomènes de sorption peuvent être pris en compte dans les étapes de modélisation-simulation.

La localisation de l'ensemble de ces investigations est influencée par l'analyse de l'existant du site. Il se peut que l'analyse de l'existant du site apporte peu d'information sur le sous-sol du site étudié, dans ce cas une reconnaissance du sous-sol doit être entreprise de manière systématique avant de localiser les prélèvements et essais à réaliser in situ.

Enfin la dernière étape de cette démarche permettant la modélisation et la simulation des écoulements pollués dans la zone non saturée du site étudié est scindée en deux sous-étapes. En effet, il est dans un premier temps nécessaire de choisir un outil de modélisation approprié aux phénomènes étudiés. Dans un deuxième temps, les modèles mathématiques choisis doivent être validés sur des expériences de références reflétant les phénomènes étudiés sur le site.

Ensuite, les écoulements pollués peuvent être modélisés et simulés en suivant la démarche classique de modélisation (délimitation du domaine, maillage, édition du problème, conditions initiales et aux limites, calculs et calage) en utilisant les paramètres spécifiques de la zone non saturée du site caractérisés lors de l’étape précédente. 


\section{Validation de l’outil de modélisation: expérience de F.Gandola}

\subsection{Principe de l'expérience}

La méthodologie de laboratoire développée par F. Gandola ${ }^{3}$ repose sur la visualisation des phénomènes d'infiltration réalisée en condition d'axisymétrie, en utilisant simultanément un traceur et une condition d'éclairage en lumière bleu. Cette technique permet de suivre l'évolution du front d'humectation durant l'infiltration. Les principaux avantages de cette méthode résident dans la non intrusivité d’objets susceptibles de perturber l'écoulement (sondes TDR,...).

\subsection{Protocole}

Cette expérience nécessite un dispositif particulier détaillé sur la figure 3, concernant le matériau, la solution infiltrée et le système de mesure.

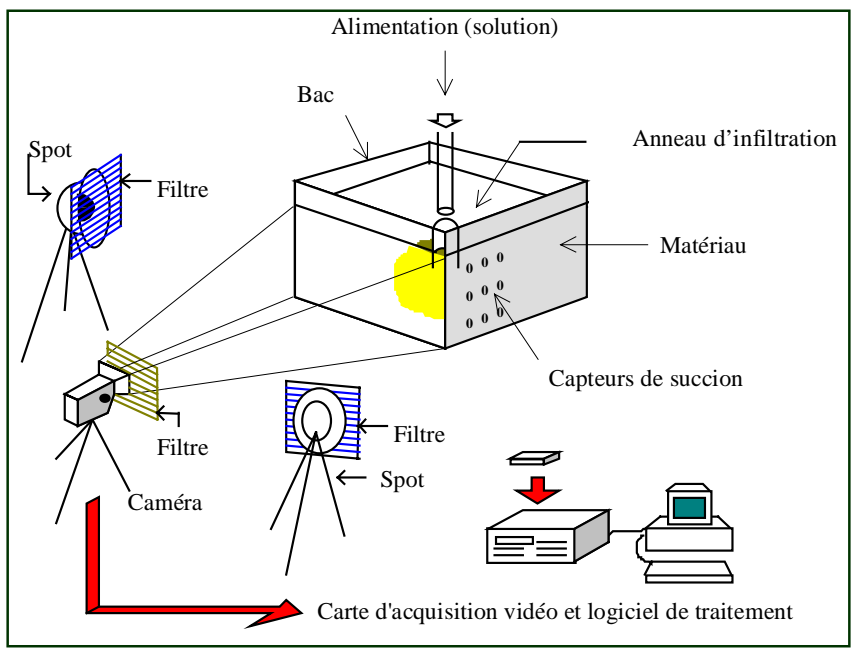

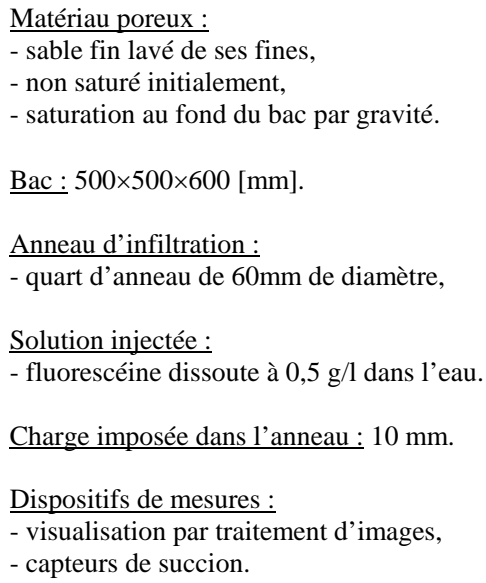

Figure 3. Dispositif expérimental.

Le système de mesure concerne les teneurs en eau déterminée par analyse d'image sur une face, ainsi que les succions en différents horizons et sur différentes verticales du massif sur une deuxième face et ceci sur une durée d'infiltration (720s) et de drainage (720s à 1320s).

\subsection{Résultats expérimentaux}

Les images et les mesures de succions sont présentées sur la figure 4. L'analyse d'image (A) montre bien l'évolution du bulbe infiltré au cours du temps avec une part importante des phénomènes de succion au début de l'infiltration (déplacements horizontaux). Au cours de l'expérience, les forces gravitaires prennent de plus en plus d'importance (déplacements verticaux). Par ailleurs, les mesures de succions (B) permettent également l'observation du front d'humectation qui est identifié par une chute de pressions. 
A
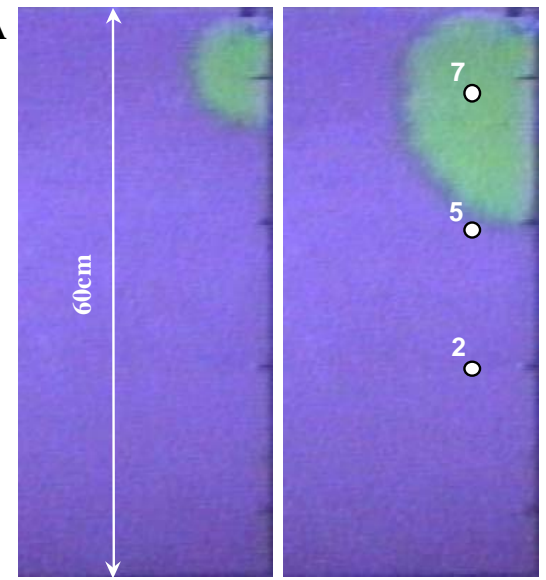

b) $360 \mathrm{~s}$
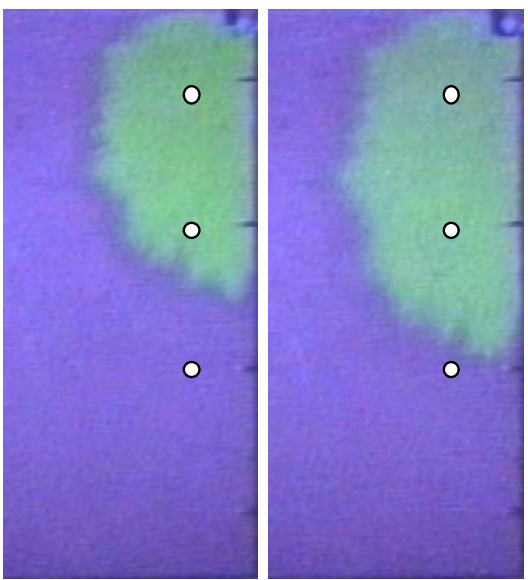

d) $1320 \mathrm{~s}$
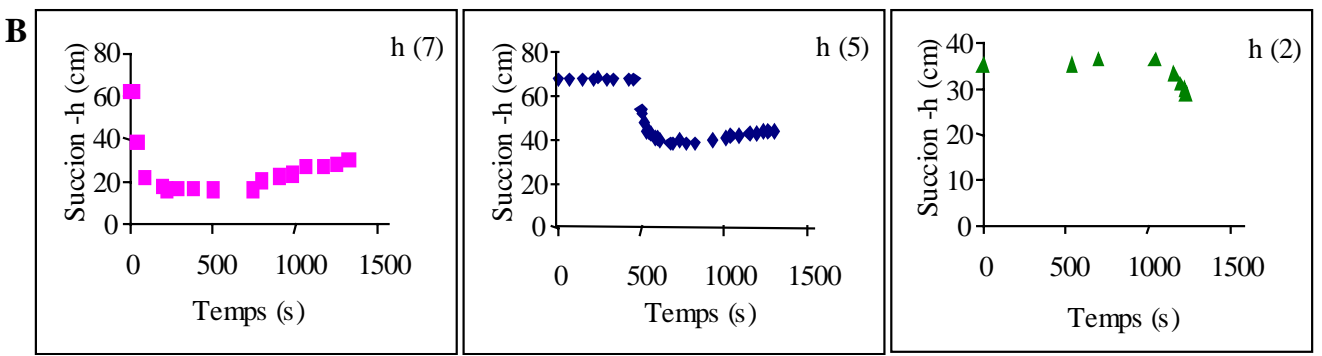

Figure 4. Résultats expérimentaux : visualisation (A), mesures de succion (B).

Il serait intéressant de vérifier que les modèles mathématiques reliant la conductivité hydraulique et la succion sont capables de représenter les résultats de cet essai original qui ne perturbe pas l'écoulement.

\subsection{Hypothèse de modélisation}

L'outil de modélisation numérique doit permettre de travailler avec les modèles mathématiques choisis au préalable, en milieux non saturés, en régime transitoire et en conditions axisymétriques. Le choix de cet outil s'est arrêté sur le logiciel Feflow $\left(G . D i e r s c h^{4}\right)$ utilisant la méthode des éléments finis. Les écoulements en milieu poreux non saturé sont résolus par l'équation non linéaire de Richards à l'aide de modèles paramétriques tels que celui de Van Genuchten ${ }^{5}$ :

$$
\begin{aligned}
& S e=\frac{\left(\theta-\theta_{r}\right)}{\left(\theta_{s}-\theta_{r}\right)}=\left(1+(\alpha \psi)^{n}\right)^{-m} \\
& \frac{K}{K_{s}}=\frac{\left[1-(\alpha \psi)^{m \cdot n}\left(1+(\alpha \psi)^{n}\right)^{-m}\right]^{2}}{\left(1+(\alpha \psi)^{n}\right)^{m / 2}}
\end{aligned}
$$

Selon le degré de saturation, $\theta$ la teneur en eau volumique ( $\theta \mathrm{r}$ résiduelle et $\theta$ s à saturation), $\mathrm{K}$ la conductivité hydraulique (Ks à saturation), $\psi$ la succion et $\alpha, \mathrm{n}, \mathrm{m}$ des paramètres de formes. 


\subsection{Paramétrage}

L’identification des paramètres à implanter dans le modèle numérique est réalisée à partir du protocole défini par F.Gandola et concerne la caractérisation du matériau, des conditions initiales et des conditions aux limites.

Le matériau est caractérisé par profils de porosité (50\%) et de teneur en eau volumique initiale (5\% en haut du bac à $40 \%$ au fond du bac). La conductivité hydraulique à saturation est définie lors de son essai et est de l'ordre du millimètre par seconde ${ }^{3}$. Les paramètres de formes $\alpha, \mathrm{n}$ et $\mathrm{m}$ du modèle de Van Genuchten sont définis par les mesures de succions à l'équilibre hydrostatique avant essai, couplées aux mesures de teneur en eau volumique. Les conditions initiales concernent l'état de saturation du matériau avant l'infiltration. Le profil de teneur en eau volumique défini par les essais est donc implanté tel quel dans le paramétrage des conditions initiales. Les conditions aux limites sont matérialisées par une charge constante imposée dans l'anneau d'infiltration $(10 \mathrm{~mm})$ sur la période d'infiltration (0s à $720 \mathrm{~s})$. Celle-ci devient nulle à partir de la phase de drainage (720s à 1320s).

\subsection{Résultats de simulation}

Les résultats de simulation sont comparés aux résultats expérimentaux (Figure 5). Les conditions initiales d'humidité du matériau sont représentées par la couleur blanche, tandis que l'assombrissement de la coloration du bulbe indique une augmentation du degré de saturation. Le front d'humectation simulé correspond, en terme de forme et de position, au front d'humectation expérimental. Le bulbe est fortement saturé en eau lors de la phase d'infiltration mais s'homogénéise lors de la phase de drainage avec une désaturation du bulbe près de l'anneau où l'alimentation est stoppée.

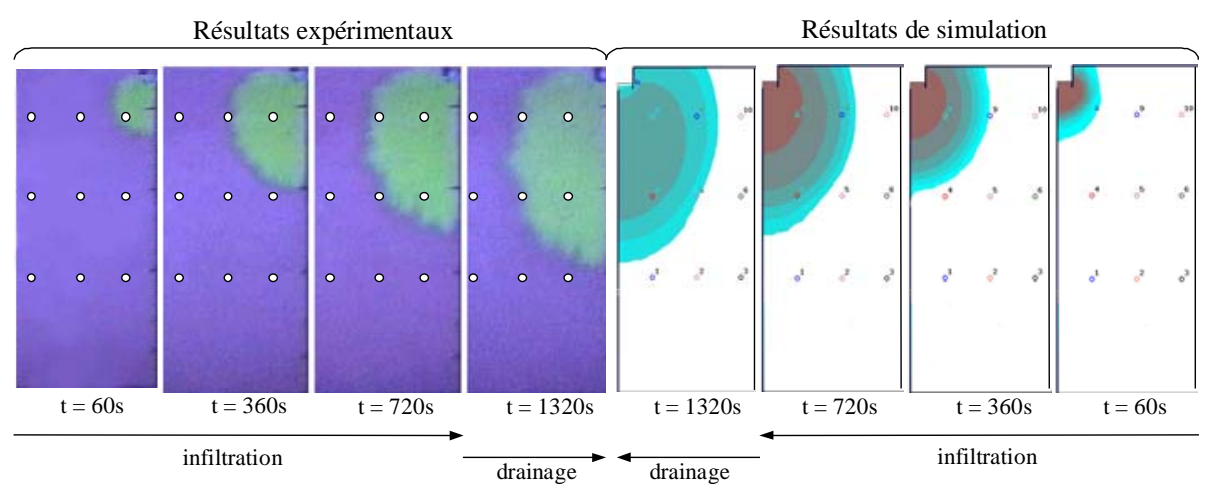

Figure 5. Comparaison des résultats simulés aux résultats expérimentaux.

Par ailleurs, la comparaison mesure-simulation des pressions aux différents points indiqués sur la figure 5 est effectuée. Une chute de pressions lors du passage du front d'humectation est obtenue tout comme les mesures de succions expérimentales réalisées par tensiométrie. 


\section{$\underline{\text { 4.Conclusion et perspectives }}$}

La démarche de travail proposée permet de caractériser et modéliser les écoulements pollués dans un sol non saturé en mêlant les informations existantes aux données d'investigation. En outre, elle est établie de manière à conforter les hypothèses initiales de travail et à maîtriser les résultats de simulation. L'étape de validation de l'outil de modélisation numérique sur une expérience d'infiltration a montré des résultats numériques proches des résultats expérimentaux. Ceci conforte le choix du modèle avant de réaliser la phase de modélisation-simulation du transport de contaminant dans la zone non saturée du site d'étude. Ceci permettra par la suite, de quantifier la part de flux contaminé pouvant atteindre le système fluvial.

\section{Références}

1 Serrano S. (1997). Hydrology, An integrated treatment of surface, subsurface and contaminant hydrology. 219-223, 386-436.

2 Richards L.A. (1931). Capillary conduction of liquids through porous medium. Physics, 1, pp.319-333.

3 Gandola F., Abriak NE. and Haverkamp R. (1999). Fluorescence appliquée à la caractérisation des phénomènes d'infiltration dans un milieu poreux non saturé : évaluation des risques de pollution. J. Chim.Phys.Bio., Vol. 96 No. 3, p 364.

4 Diersch H-J G. (1998). Feflow, finite-element simulation system for modeling groundwater flow, contaminant mass processes. WAZY research Ltd.

5 Van Genucthen M.T. (1980). A closed form equation for predicting the hydraulic conductivity of unsaturated soils. Soil Sci. Soc. Am. J., 44 pp892-898. 